\title{
ON THE MINIMUM HOP COUNT AND CONNECTIVITY IN ONE-DIMENSIONAL AD HOC WIRELESS NETWORKS
}

\author{
NELSON ANTUNES, GONÇALO JACINTO, AND ANTÓNIO PACHECO
}

\begin{abstract}
This paper investigates connectivity in one-dimensional ad hoc networks by means of the distribution of the minimum hop count between source and destination nodes. We derive the exact probability distribution of the minimum hop count from the location density of relay nodes in the multihop path selected with the Most Forward within Radius (MFR) scheme. The probability that the source and destination nodes are connected (provided by Ghasemi and Nadser-Esfahani (2006)) can be obtained by summing the probability masses for each possible value of the minimum hop count, which provides new insights to the connectivity probability. Numerical results show the effect of the number of nodes and the transmission range on the minimum hop count.
\end{abstract}

Keywords: Ad hoc network, one-dimensional, connectivity probability, Minimum Hop count, Poisson randomization

\section{INTRODUCTION}

In an ad hoc multi-hop network, the functionality of the network critically depends on its connectivity properties. These depend on the number of nodes, their transmission ranges, and spatial distribution resulting from the mobility pattern. A common approach when evaluating connectivity is to test if all adjacent nodes are within the transmission range from each other, which implies that there exists some multihop path between any two nodes.

Several studies of connectivity between two nodes and for the entire network in onedimensional ad hoc networks have appeared in the literature. One of the first results for the connectivity probability of a finite set of nodes uniformly distributed in a finite interval was given by Desai and Manjunath (2002). Foh and Lee (2004) and Foh et al. (2005) derived closed form approximation formulas for the connectivity between two nodes when relay nodes are uniformly and non-uniformly distributed, respectively. Li et al. (2006) investigated an empirical approach for the distribution of the maximum distance between adjacent nodes assuming non-identically distributed locations. Ghasemi and Nader-Esfahani (2006) obtained 
the probability that the one-dimensional ad hoc network is composed of at most $C$ clusters. Other issues of interest are the number of nodes and the transmission range to ensure that the network is connected (see, e.g., Santi and Blough (2003) and Bettstetter (2002)).

It is important to ensure that a source and a destination node are connected with a high probability. In addition, it is of interest to be able to provide information on the length of paths (number of hops) between the source and destination nodes. In this paper we derive the distribution of the minimum hop count between a source and a destination node in a one dimensional ad hoc network and show that it can be related with the connectivity probability. This extends the existing results for the connectivity probability (derived in Gasemi and Nader-Esfahani (2006) with a different approach) through its decomposition as the sum of the probabilities for values of the minimum hop count.

We consider a one dimensional ad hoc network where the source and destination nodes are located at the edge of the network and a fixed number of relay nodes are uniformly distributed between them. This spatial distribution of nodes arises when nodes move according to a Random Walk or a Random Direction mobility scheme over the region of interest (Camp, Boleng and Davies (2002)). We derive an exact formula for the probability distribution of the minimum hop count based on the location density of relay nodes in the multihop path selected with the Most Forward within the Radius (MFR) scheme (Takagi and Kleinrock (1984)). Starting from the source node, each successive relay node in the MFR path is selected so that it provides the greatest forward progress toward the destination node within the fixed transmission range. Therefore, the minimum hop count is given by the number of hops in the MFR path.

The study of the distribution of the minimum hop count in a one-dimensional ad hoc network (e.g., a vehicular ad hoc network built along a road in a city environment or an ad hoc network along an attack route in battlefields) has also applications in other scenarios where nodes are in a line. This is the case for dense two dimensional ad hoc networks where routes are approximately straight line segments (Hyytia et al. (2005)). Hence, the transmission between a source and a destination node constitutes a multihop path on the line.

This paper is organized as follows. Section 2 describes the considered ad hoc network. In Section 3 , a recursive formula is presented to compute the connectivity probability with the minimum hop count. Section 4 provides an alternative way to derive the probability presented 
in Section 3 using a randomization method. In Section 5 we present some numerical results and, finally, we state some conclusions in Section 6.

\section{MODEL DESCRIPTION}

We consider a multihop ad hoc network with a source node, a destination node, and $N$ relay nodes between them. Let $X_{i}, i=1,2, \ldots, N$, denote the location of relay node $i$, and $X_{0}=0$ and $X_{N+1}=L$ denote the location of the source and destination nodes, respectively. We assume that $\left\{X_{i}\right\}_{i=1}^{N}$ are independent and uniformly distributed random variables on $(0, L)$ and let $X_{(i)}, i=0,1, \ldots, N+1$, denote the location of the $i$ th node from the origin, i.e.,

$$
0=X_{(0)}<X_{(1)}<\ldots<X_{(N+1)}=L .
$$

Given a fixed transmission range $0<R<L$ equal for all nodes, nodes $i$ and $j$ are connected if $\left|X_{i}-X_{j}\right| \leq R$. If the source and destination nodes are connected, i.e., $X_{(i+1)}-X_{(i)} \leq R$, $i=0,1, \ldots, N$, the MFR multihop path is given by $Y^{M}=\left(Y_{1}, Y_{2}, \ldots, Y_{M}\right)$ where

$$
\begin{aligned}
Y_{1}=\max \left\{X_{(i)}: X_{(i)} \leq R\right\}, Y_{2}=\max \left\{X_{(i)}: X_{(i)} \leq Y_{1}+R\right\}, \ldots, \\
Y_{M}=\max \left\{X_{(i)}: X_{(i)} \leq Y_{M-1}+R\right\}
\end{aligned}
$$

with $Y_{M-1}+R<X_{N+1} \leq Y_{M}+R$ (see Figure 1), and $M$ is called the minimum hop count.

The minimum number of hops that may be needed to connect the source and destination nodes is denoted by $K=\lceil L / R\rceil-1$. To simplify the exposition we define $K$ zones between the source and destination nodes denoted by $Z_{1}, Z_{2}, \ldots, Z_{K}$, with zone $i$ starting at location $Z_{i}^{-}=L-R(K+1-i)$ and ending at $Z_{i}^{+}=i R$, with length $Z=(K+1) R-L$. From Figure 1 , the minimum hop count is $K$ if there exist relay nodes in the zones $Z_{1}, \ldots, Z_{K}$ in such way that

$$
Z_{1}^{-}<Y_{1}<Z_{1}^{+}, \quad Z_{2}^{-}<Y_{2}<Y_{1}+R, \ldots, \quad Z_{K}^{-}<Y_{K}<Y_{K-1}+R .
$$

On the other hand, the maximum number of hops that may be needed to connect the source and destination nodes is $2 K$, which occurs if the zones $Z_{i}, i=1,2, \ldots, K$, are empty, the first relay node in the MFR multihop path is before $Z_{1}^{-}$, and between each $Z_{i}^{+}$and $Z_{i+1}^{-}$there 
exists two relay nodes in the multihop path with the last relay node after $Z_{K}^{+}$, such that

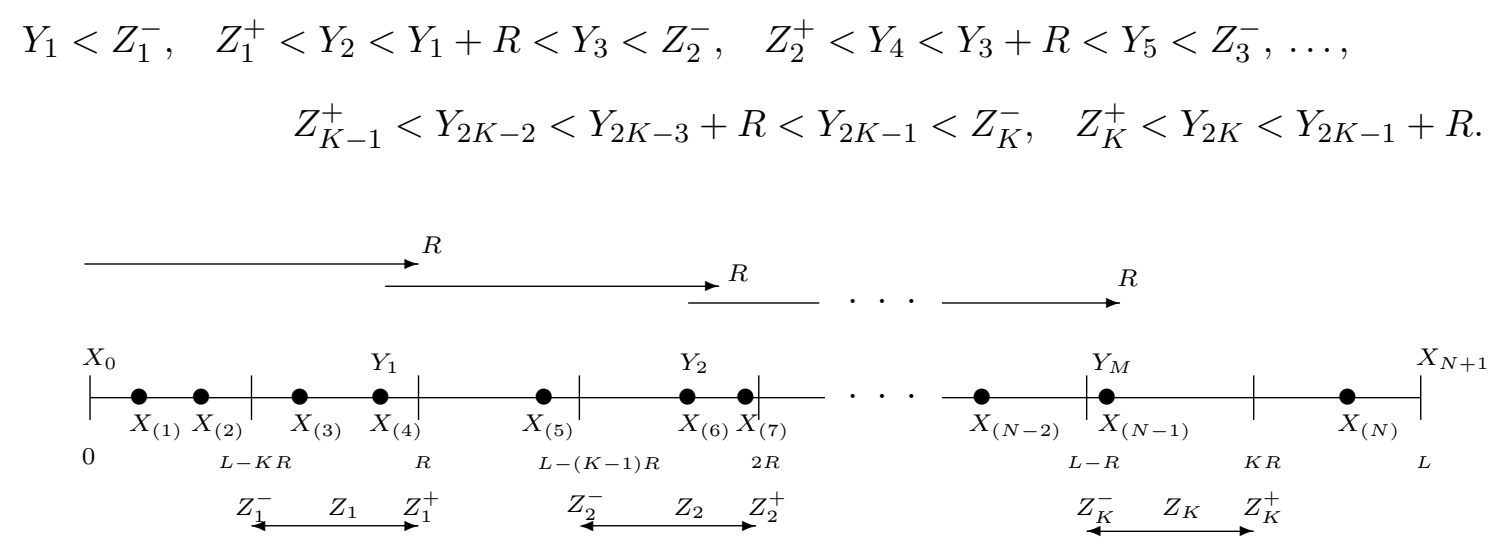

Figure 1. Minimum hop count.

\section{A RECURSIVE FORMUlA}

In this section we derive a recursive formula to compute the probability distribution of the minimum hop count.

The probability density of the location of the first hop in the MFR path being $t$ and the order of the associated relay node being $i$ is

$$
f^{(i)}(t \mid N, R, L)=\frac{i}{L}\left(\begin{array}{c}
N \\
i
\end{array}\right)\left(\frac{t}{L}\right)^{i-1}\left(1-\frac{R}{L}\right)^{N-i}, \quad 0<t \leq R, i=1, \ldots, N .
$$

This results from the marginal density location of $X_{(i)}$ (see, e.g., Kulkarni (1995, page 209, Eq. (5.78))) taking into account that the last $N-i$ ordered nodes have to be located on $(R, L)$. Given the location $t$ of the first hop and the order $i$ of the associated relay node, the locations of the last $N-i$ ordered relay nodes are independent and uniformly distributed on $(R, L)$.

In addition, the probability that the minimum hop count is $m$ given that the location and the order of the first hop are $t$ and $i$, respectively, is equal to the probability that the minimum hop count is $m-1$ between a pair of nodes located at the endpoints of interval $[R, L]$ where: the source is at $R$ and has transmission range $t$; and, $N-i$ relay nodes, each with transmission range $R$, are uniformly distributed on $(R, L)$. This fact can be used to derive a recursive formula to compute the probability distribution of the minimum hop count, as it will be explained. 
We let $p(m \mid n, r, l)$ denote the probability that the minimum hop count is $m$ when the distance between the source and destination nodes is $l$, the transmission range of the source is $r$, and there are $n$ relay nodes, each with transmission range $R$, uniformly placed between the source and destination nodes. Then,

$$
\begin{aligned}
& p(m \mid n, r, l)= \\
& \begin{cases}\int_{0}^{r} \sum_{i=1}^{n} f^{(i)}(t \mid n, r, l) p(m-1 \mid n-i, t+R-r, l-r) d t & r<l, k \leq m \leq \min (2 k, n), \\
1 & r \geq l, m=0, \\
0 & \text { otherwise, }\end{cases}
\end{aligned}
$$

where $k=1+\lfloor(l-r) / R\rfloor$, and the probability that the minimum hop count is $m$ is then given by $p(m \mid N, R, L)$.

\section{Poisson RANDOMization}

The recursive formula given by equation (1) suffers from combinatorial problems and its computation is hard even for moderate values of $m$ and $n$. In this section we show that the use of Poisson randomization, an exact method originally proposed by Domb (1952), allows for the derivation of an elegant formula without the mentioned drawbacks.

Our use of Poisson randomization consists in randomizing the distribution of relay nodes, and as a consequence of the parameter $N$, by assuming that relay nodes are distributed on $(0, L)$ according to a Poisson process with a fixed rate, say $\lambda$. This assumption decouples the relay nodes in the sense that the number of relay nodes located in disjoint intervals are independent. Moreover, by conditioning on the number of nodes that lie on $(0, L)$, the assumption that the relay nodes are uniformly distributed on $(0, L)$ pops up. As a result of these facts, the probability that the minimum hop count $(M)$ is equal to $m$ can be explicity derived with little effort. Moreover, by viewing it as an instance of the total probability law formula in the form

$$
P(M=m)=\sum_{n=m}^{\infty} e^{-\lambda L} \frac{(\lambda L)^{n}}{n !} P(M=m \mid N=n),
$$

we can identify the probability that the minimum hop count is equal to $m$ when the number of relay nodes is $n, P(M=m \mid N=n), n \in \mathbb{N}$. It turns out that this leads to a more efficient 
way to compute the minimum hop count distribution for a fixed number of relay nodes than the one described through (1).

The next result characterizes the exact probability distribution of the minimum hop count given the number of relay nodes between the source and destination nodes, which is a consequence of the successful use of Poisson randomization.

Theorem 1. Given that the relay nodes are uniformly distributed on $(0, L)$, the probability distribution of the minimum hop count is given by

$$
\mathbb{P}(M=m \mid N=n)=\int_{S_{m}} \frac{n !}{(n-m) ! L^{n}}\left(L-m R+y_{m}\right)^{n-m} d y_{m} d y_{m-1} \ldots d y_{1},
$$

with $K \leq m \leq \min (2 K, n)$ and where, with $y_{0}=0$ and $y_{m+1}=L$,

$$
S_{m}=\left\{\left(y_{1}, y_{2}, \ldots, y_{m}\right) \in(0, L)^{m}: y_{1}<R, y_{j-2}+R<y_{j} \leq y_{j-1}+R, 2 \leq j \leq m+1\right\} .
$$

Proof. We assume that the relay nodes are distributed on $(0, L)$ according to a Poisson process with rate $\lambda$. In this setting, the probability that the the minimum hop count is $m$ is

$$
\mathbb{P}(M=m)=\int_{S_{m}} \lambda e^{-\lambda\left(R-y_{1}\right)} \prod_{i=1}^{m-1} \lambda e^{-\lambda\left(y_{i}+R-y_{i+1}\right)} d y_{m} d y_{m-1} \ldots d y_{1} .
$$

Multiplying equation (3) by $e^{\lambda L}$, we obtain

$$
\begin{aligned}
e^{\lambda L} \mathbb{P}(M=m) & =e^{\lambda L} \int_{S_{m}} \lambda^{m} e^{-\lambda\left(m R-y_{m}\right)} d y_{m} d y_{m-1} \ldots d y_{1} \\
& =\int_{S_{m}} \lambda^{m} \sum_{n=0}^{\infty} \frac{\lambda^{n}}{n !}\left(L-m R+y_{m}\right)^{n} d y_{m} d y_{m-1} \ldots d y_{1} \\
& =\sum_{n=m}^{\infty} \frac{(\lambda L)^{n}}{n !} \frac{n !}{(n-m) ! L^{n}} \int_{S_{m}}\left(L-m R+y_{m}\right)^{n-m} d y_{m} d y_{m-1} \ldots d y_{1}
\end{aligned}
$$

where the change between the sum and the integral follows by the dominated convergence theorem. On the other hand, conditioning on the value of $N$, which is Poisson distributed with mean $\lambda L$, the total probability law produces

$$
e^{\lambda L} \mathbb{P}(M=m)=\sum_{n=m}^{\infty} \mathbb{P}(M=m \mid N=n) \frac{(\lambda L)^{n}}{n !} .
$$

Since the coefficients of $(\lambda L)^{n} / n$ ! in the previous two expressions for $e^{\lambda L} \mathbb{P}(M=m)$ must match, the result follows. 
We remark that the integrand function in (2) is the probability density function of the location of the relay nodes in the MFR path $\left(Y^{M}\right)$. The next results show that, for some values of $M$, there exists a closed form formula for the connectivity probability with the minimum hop count.

Corollary 1. The probability that the minimum hop count is equal to $K$ is given by

$$
\mathbb{P}(M=K \mid N=n)=\sum_{i=K}^{n}\left(\begin{array}{l}
n \\
i
\end{array}\right)\left(\frac{Z}{L}\right)^{i}\left(1-\frac{Z}{L}\right)^{n-i}
$$

for $K=\lceil L / R\rceil-1 \leq n$.

Proof. From Theorem 1, we have

$$
\begin{aligned}
\mathbb{P}(M=K \mid N=n) & =\frac{n !}{(n-K) ! L^{n}} \int_{Z_{1}^{-}}^{Z_{1}^{+}} \int_{Z_{2}^{-}}^{y_{1}+R} \ldots \int_{Z_{K}^{-}}^{y_{K-1}+R}\left(L-K R+y_{K}\right)^{n-K} d y_{K} d y_{K-1} \ldots d y_{1} \\
& =\frac{n !}{(n-K) ! L^{n}} \int_{0}^{Z} \int_{0}^{s_{1}} \ldots \int_{0}^{s_{K-1}}\left(L-Z+s_{K}\right)^{n-K} d s_{K} d s_{K-1} \ldots d s_{1}
\end{aligned}
$$

by the change of variables $s_{i}=y_{i}-Z_{i}^{-}, i=1,2, \ldots, K$. Using the Binomial theorem, the results follows after some algebra.

Equation (4) is the survival function at $K$ of the binomial distribution with parameters $N$ and $Z / L$. Since a binomial random variable is stochastically increasing in the number of trials, when the success probability is kept fixed, the probability that the minimum hop count is equal to $K$ is increasing in $N$.

The probability that the minimum hop count is $K+1$ can be obtained in a similar way but now there exist other possible location zones for the hops. Closed form expressions for other values of $M$ are out of reach because of the complexity involved.

Corollary 2. The probability that the minimum hop count is equal to $K+1$ is

$$
\begin{aligned}
\mathbb{P}(M=K+1 \mid N=n)= & \sum_{i=K+1}^{n}\left(\begin{array}{c}
n \\
i
\end{array}\right)\left(\frac{L-R-Z}{L}\right)^{n-i} \\
& {\left[\sum_{j=0}^{K-1}\left(\begin{array}{c}
i \\
j
\end{array}\right)\left(\frac{Z}{L}\right)^{j}\left(\frac{R}{L}\right)^{i-j}+\left(\frac{Z}{L}\right)^{i-1}\left(\frac{i(Z-K R)-Z}{L}\right)\right] }
\end{aligned}
$$

for $K=\lceil L / R\rceil-1<n$. 
Since the set of all locations of relay nodes where the source and destination nodes are connected can be decomposed as the union of disjoint sets of the locations of nodes with respect to the minimum number of hops necessary to connect the source and destination nodes, the full connectivity probability is given by

$$
P_{c}=\sum_{m=K}^{\min (2 K, n)} \mathbb{P}(M=m \mid N=n) .
$$

\section{NumericAl RESUlts}

In this section we evaluate the effect of the number of nodes and the transmission range in the shape of the distribution of the minimum hop count. We scale all the parameters with respect to the distance between the source and destination nodes by making $L=1$. Therefore, for $1 /(k+1) \leq R<1 / k, k \in \mathbb{N}$, we have that $K=k$ and $Z=(k+1) R-1$, so that $0<Z<1 / k$. The most interesting case is when $K$ is small, since as the number of hops increases a multihop path may break frequently due to mobility of nodes (Jiang and Rao (2005)).

Figure 2 shows the connectivity probability and values of the probability function of the minimum hop count between the source and destination nodes when $R=0.32$ ( $K=3$, $Z=0.28$ ) for different values of $N$. The (full) connectivity probability is given by the sum of the probabilities of the minimum hop count being 3,4 , and 5 or more (denoted by $5+$ ) that, as can be seen from the figure, is approximately equal to the probability of the minimum hop count being 3 or 4 . As expected, with the increase of the number of relay nodes, the probability that the minimum hop count is 4 (i.e., $K+1$ ) starts to decrease and goes to zero.

In Figure 3, we consider the connectivity probability and values of the probability function of the minimum hop count between the source and destination nodes when $R=0.23$ ( $K=4$, $Z=0.15)$ for different values of $N$. Here, the connectivity probability is given by the sum of the probabilities of the minimum hop count being $4,5,6$, and 7 or more (denoted by $7+$ ) that, as can be seen from the figure, is approximately equal to the probability of the minimum hop count being 4,5 , or 6 . Moreover, the connectivity probability can be approximated by the sum of the probabilities for the two lower values of $M, 4$ and 5 , when the number of relay nodes is large. 
As the length of the intersections zones $(\mathrm{Z})$ decreases, due to the increase of $K$ or by the value of the transmission range $(\mathrm{R})$, and gets close to zero, the connectivity probability in more than $K+1$ hops stops being negligible. However, as the number of relay nodes increases its probability starts to decrease and converges to zero.

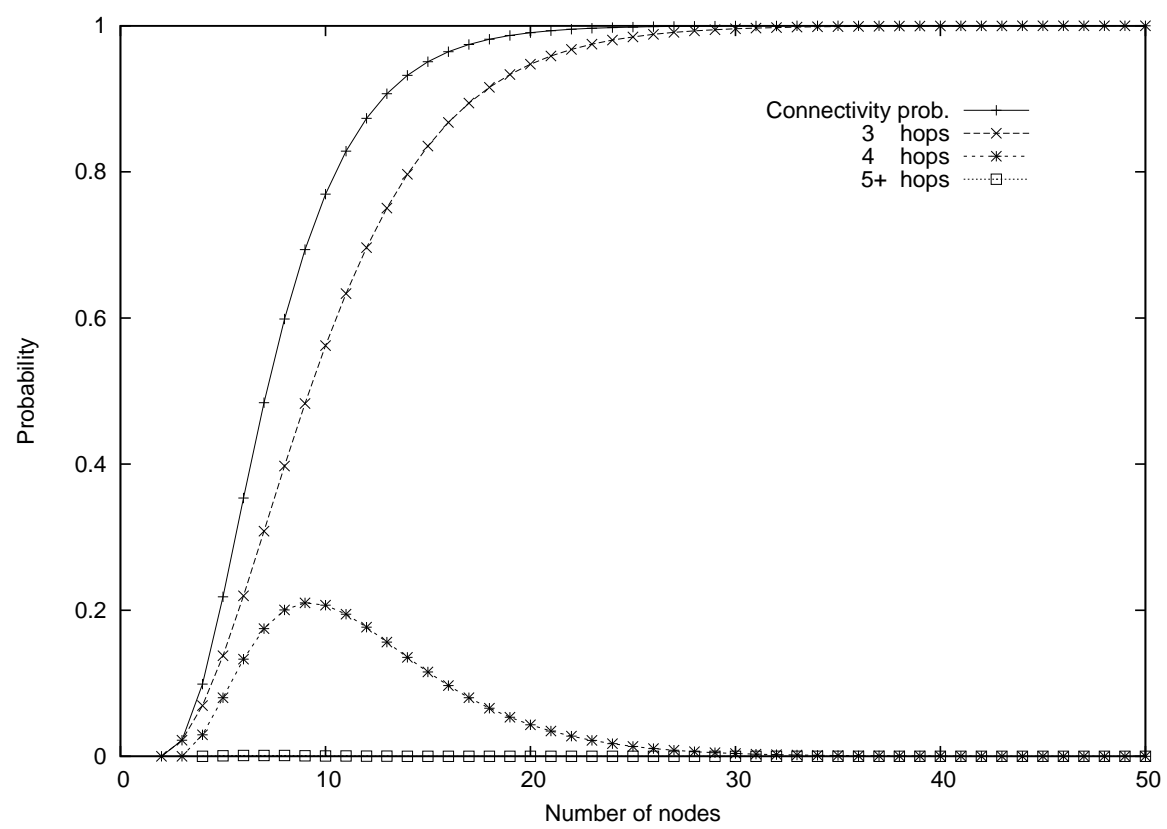

Figure 2. Probability function of the minimum hop count/connectivity probability $(L=1, R=0.32)$.

In Figure 4, we depict both probabilities that the minimum hop count is equal to $K$ and $K+1$, as a function of $R$, for $N=20$. We can see that in this setting $K$ takes values $2,3,4$, and 5 in the interval of the transmission range. In an interval for $R$ of the form $[1 /(k+1), 1 / k)$, the probability that the minimum hop count is $K$ increases with $R$, since the size of the intersection zones $(Z)$ also increases as $R$ increases.

\section{Conclusion}

We have derived the exact distribution of the minimum hop count between source and destination nodes. This makes it possible to decompose the connectivity probability, derived by Ghasemi and Nadser-Esfahani (2006), as the sum of the probability masses of the minimum hop count at each of its possible values. Through numerical experiments we have shown that 


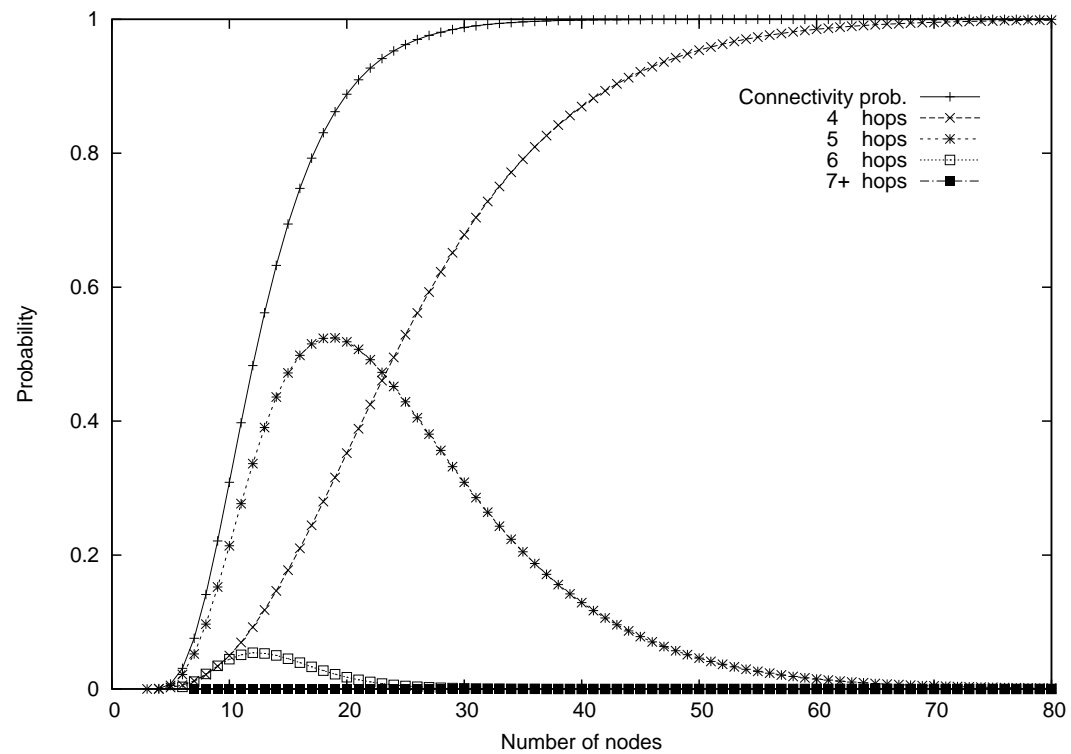

FiguRE 3. Probability function of the minimum hop count/connectivity probability $(L=1, R=0.23)$.

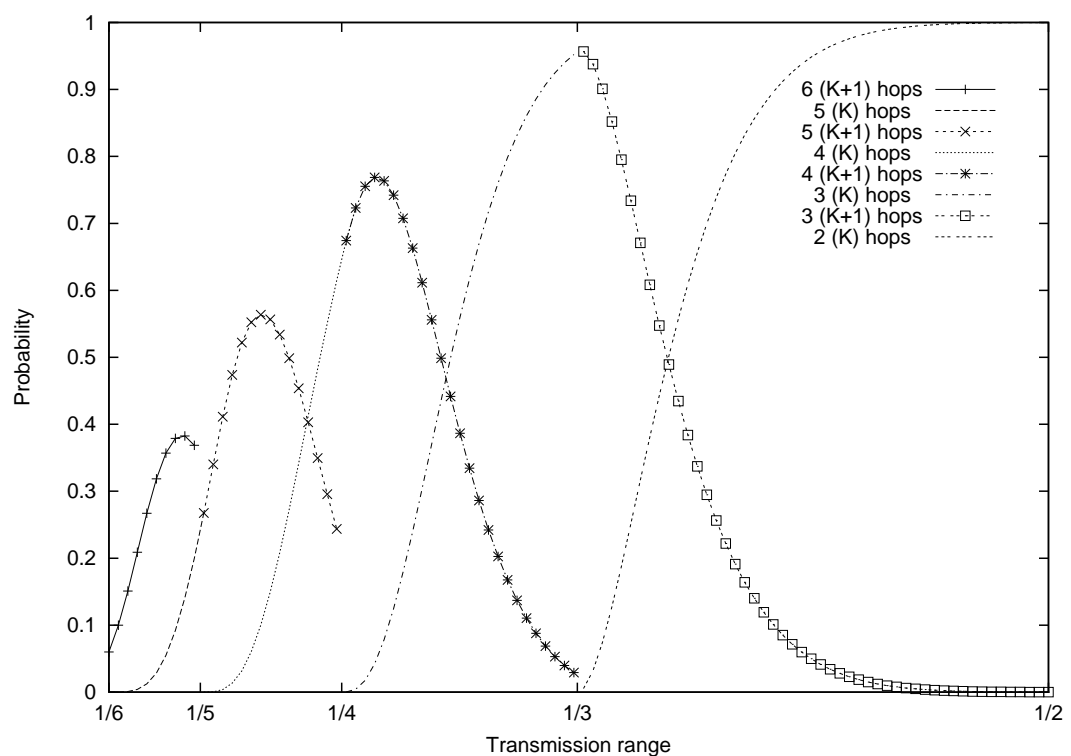

Figure 4. Probabilities of the minimum hop count being $K$ and $K+1$ ( $L=1$, $N=20)$.

under some network conditions only the the first values of the minimum hop count have contribution to the connectivity probability. 


\section{REFERENCES}

Bettstetter, C. (2002). "On the minimum node degree and connectivity of a wireless multihop network," In Proc. ACM MobiHoc, 80-91.

Camp, T., J. Boleng, and V. Davies (2002). "A survey of mobility models for ad hoc network research," Wireless Communications and Mobile Computing, 2(5):483-502.

Desai, M. and D. Manjunath (2002). "On the connectivity in finite ad hoc networks," IEEE Communications Letters, 6(10):437-439.

Domb, C. (1952). "On the use of a random parameter in combinatorial problems," Proceedings Physical Society, Sec. A, 65(1):305-309.

Foh, C.H. and B.S. Lee (2004). "A closed form network connectivity formula for one-dimensional MANETs," In Proc. IEEE ICC 2004, volume 6, pages 3739-3742.

Foh,C.H., G. Liu, B. S. Lee, B.-C. Seet, K.-J.Wong, and C.P. Fu (2005). "Network connectivity of onedimensional MANETs with random waypoint movement," IEEE Communications Letters, 9(1):31-33.

Ghasemi, A. and S. Nader-Esfahani (2006). "Exact probability of connectivity in one-dimensional ad hoc wireless networks," IEEE Communications Letters, 10(4):251-253.

Hyytia, E., P. Lassila, P. Penttinen, and J. Roszik (2005). "Traffic load in a dense wireless multihop network," In Proc. of the 2nd ACM international workshop on Performance evaluation of wireless ad hoc, sensor, and ubiquitous networks, pages 9-17.

Jiang, S. and J. Rao (2005). "A prediction-based link availability estimation for routing metrics in MANETs," IEEE/ACM Transactions on Networking, 13(6):1302-1312.

Kulkarni, V. G. (1995). "Modeling and Analysis of Stochastic Systems," Chapman and Hall.

Li, J., L.L.H. Andrew, C.H. Foh, M. Zukerman, and M.F. Neuts (2006). "Meeting connectivity requirements in a wireless multihop network," IEEE Communications Letters, 10(1):19-21.

Santi, P. and D.M. Blough (2003). "The critical transmitting range for connectivity in sparse wireless ad hoc networks," IEEE Transactions on Mobile Computing, 2(1):25-39, Jan.-Mar.

Takagi, H. and L. Kleinrock (1984). "Optimal transmission ranges for randomly distributed packet radio terminals," IEEE Transactions on Communications, 32(3):246-57.

Toh, C.K. (2002). "Ad Hoc Mobile Wireless Networks: Protocols and Systems," Prentice Hall. 
Nelson Antunes

CEMAT and FCT da Universidade do Algarve,

Campus de Gambelas, 8005-139 Faro, Portugal

email: nantunes@ualg.pt

Gonçalo Jacinto

CIMA-EU and Department of Mathematics of Évora University

Colégio Luís Verney, Rua Romão Ramalho, 59, 7000-671, Évora, Portugal

email: gjcj@uevora.pt

António Pacheco

CEMAT and Department of Mathematics, Instituto Superior Técnico - TU Lisbon,

Av. Rovisco Pais, 1049-001, Lisboa, Portugal

email: apacheco@math.ist.utl.pt

This research was supported in part by Programa Operacional "Ciência, Tecnologia, Inovação" (POCTI) of the Fundação para a Ciência e a Tecnologia (FCT), cofinanced by the European Union fund FEDER, and the projects POSC/EIA/60061/2004 and Euro-FGI. 